\title{
Probing the Structural Organization of Virions and Genomic Ribonucleoprotein Complexes from Type B Influenza Virus by Cryo-electron Microscopy
}

\author{
Neetu M. Gulati ${ }^{1}$, John R. Gallagher ${ }^{1}$, Dustin M. McCraw ${ }^{1}$ and Audray K. Harris ${ }^{1 *}$ \\ ${ }^{1 .}$ Laboratory of Infectious Diseases, National Institute of Allergy and Infectious Diseases, National \\ Institutes of Health, Bethesda, MD, USA. \\ * Corresponding author: harrisau@mail.nih.gov
}

Influenza viruses cause millions of infections each year. Unlike influenza A viruses, influenza B infection appears restricted to humans and no zoonotic reservoirs have been identified [1]. Although numerous structural studies of influenza A viruses have been reported [2-4], less is known about the structural organization of influenza B. Additionally, the structural organizations of packaged and unpackaged segmented influenza B genomic ribonucleoprotein complexes (RNPs) have not been studied in detail [5]. The lack of such structural information hinders studies on how adaption to different culture systems could affect the morphologies of influenza B viruses and their genomic RNPs along with viral entry, assembly and transmission. Here, we used cryo-electron microscopy (cryo-EM) and image analyses to probe the structural organization of influenza type B viruses and their genomic RNPs.

Influenza B viruses isolated from MDCK cells (Figure 1a,c) and chicken eggs (Figure 1d) were both observed to be roughly spherical in shape when imaged by negative-stain or cryo-EM (Figure 1c,d). Interestingly, we were able to separate smaller, irregular particles that appeared to be membrane blebs (Figure 1b) from intact virions (Figure 1c). When analyzed by cryo-EM, influenza B virions were pleiomorphic but approached spherical morphologies (Figure 1d-f). Virions $(\mathrm{N}=695)$ had an average major axis of $107 \mathrm{~nm}$ in length (40-275 nm range) and an average minor axis of $94 \mathrm{~nm}$ in length (36-169 $\mathrm{nm}$ range). The average virion diameter was $100 \mathrm{~nm}$ and the majority of virions had an aspect ratio below 1.2 with an enclosed area of less than $10000 \mathrm{~nm}^{2}$. These results indicate that the majority of influenza type $\mathrm{B}$ viruses are approximately spherical when grown in either MDCK or egg-adapted. This may indicate that morphological adaptions and transmission of influenza B do not follow the dogma established for influenza A virus. For type A, spherical virions are considered to be less transmissible than filamentous virions, which tend to dominate MDCK-grown virus preparations as opposed to more spherical virions from egg-adaption [6].

Cryo-electron tomography revealed that RNP filaments were packaged in viruses (Figure 2a-d) and were also present in an unpackaged, isolated state (Figure 2e-h). Lateral views of RNPs suggested a variable, double-helical structure (Figure 2a,e-h). Apical views of the RNPs within virus (Figure 2b-d) revealed a $7+1$ arrangement of RNPs with a central RNP surrounded by seven others, presented as C-shaped densities (Figure 2c, 2d). Interestingly, we observed densities that appeared to bridge RNPs (Figure 2d, arrows). One speculation is that these densities could represent macromolecular interactions among RNPs involved in the selective packaging of eight RNPs in virions [7]. Rotational averages (e.g. Figure 2, inset), of computationally extracted RNP sections (Figure 2d) indicate the filament thickness varied from 12-19nm, consistent with measurements for side views of isolated filaments which had an average thickness of 14.2 $\mathrm{nm}(\mathrm{N}=42$; Figure $2 \mathrm{e}-\mathrm{h})$. In some instances, helical repeats between 20-25 nm on isolated RNPs were observed (Figure 2h, arrows). The double-helical organization of RNPs and their 7+1 arrangement inside influenza B virions imply that the mechanisms for the assembly of RNPs and their incorporation into budding virions are conserved between influenza B and A viruses [7-9]. Packaging of all 8 distinct RNP 
genomic segments is imperative for influenza fitness, and the structural features necessary for proper RNP and virus assembly is the subject of future work [10].

\section{References:}

[1] C Zhao et al., Trends Microbiol 21 (2013), p. 181.

[2] A Harris et al., Proc Natl Acad Sci U S A 103 (2006), p. 19123.

[3] AK Harris et al., Proc Natl Acad Sci U S A 110 (2013), p. 4592.

[4] DM McCraw et al., Sci Rep 8 (2018), p. 10342.

[5] G Katz et al., PLoS One 9 (2014), p. e88288.

[6] PJ Campbell et al., J Virol 88 (2014), p. 3802.

[7] T Noda et al., Nat Commun 9 (2018), p. 54.

[8] JR Gallagher et al., J Struct Biol, 10.1016/j.jsb.2016.12.007(2016), p.

[9] W Zheng and YJ Tao, FEBS Lett 587 (2013), p. 1206.

[10] The authors acknowledge support from the Intramural Research Program of NIAID/NIH and V. Nair and E. Fischer at the NIAID Research Technologies Branch for aid in cryo-EM data collection.
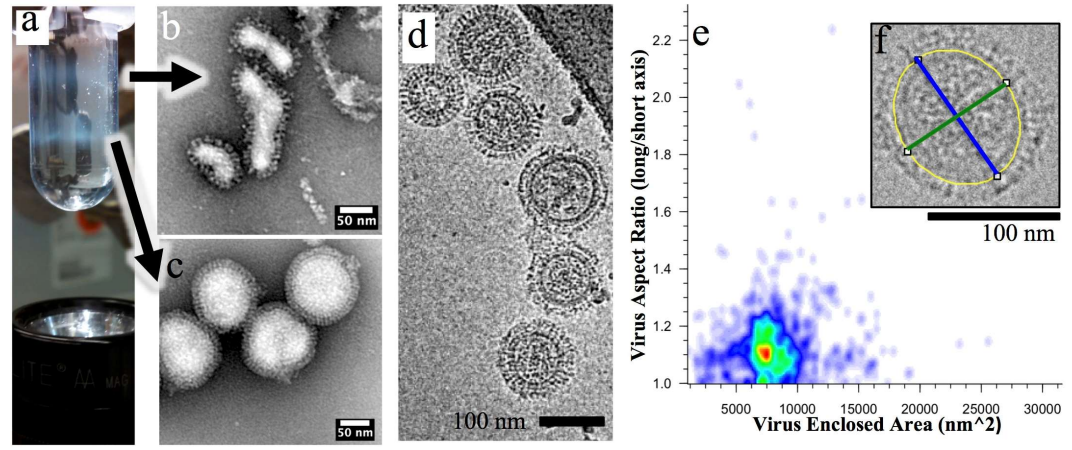

Figure 1. Analysis of influenza $B$ virus (B/Lee/40) by electron microscopy. (a) Sucrose gradient purification of virus from MDCK cells. (b, c) Negative-staining electron microscopy of top (b) and bottom (c) bands. (d) Cryo-EM of egg-adapted virus. (e) Analysis of virus morphology by heat-map analysis using measurements of aspect ratios derived from long and short axes and enclosed area from cryo-EM images of virus particles ( $\mathrm{N}=695)$. (f) Example of a measurement of virion morphology using an elliptical model using major (blue) and minor (green) axes.
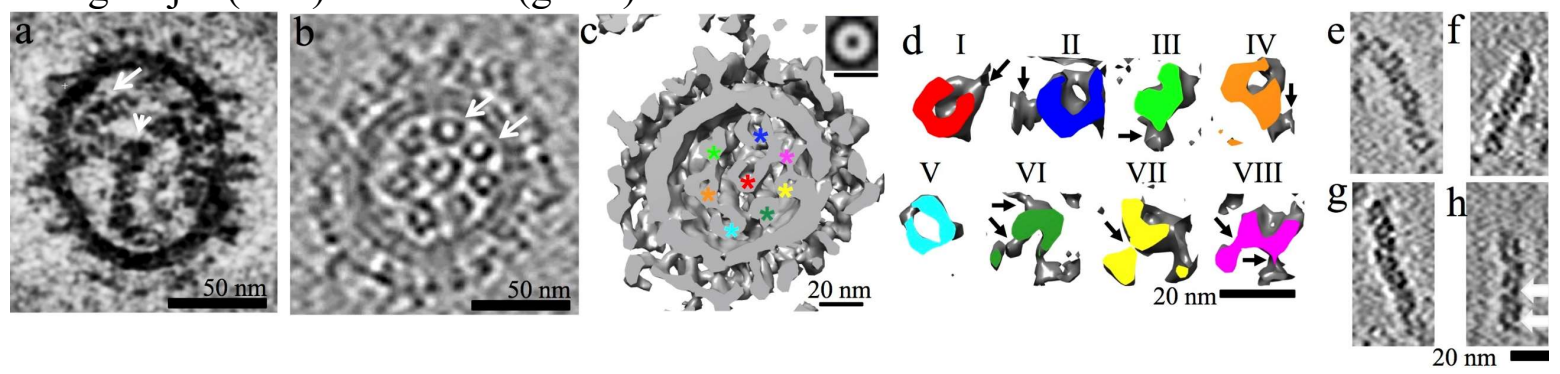

Figure 2. Analysis of RNP organization of influenza B viruses by cryo-electron tomography. (a, b) Slices through tomograms of two different virions imaged with the long axes of RNPs oriented to give approximately side-views (panel a) and top-views (panel b) of the RNP filaments (arrows). (c) Virion in panel $b$ shown as a surface rendering with eight RNPs denoted by colored asterisks. Inset, rotational average of the central RNP (red asterisk). (d) Surface rendering of the eight RNP sections from panel c colored for RNPs I-VIII. (e-h) Gallery of slices through tomograms of isolated RNPs. Arrows (panel h) indicate apparent repeats of the RNP filament. Panel a is from egg-adapted B/Lee/40 and panel b-d is from MDCK of strain B/Victoria/304/2006. 